2020-07-31

\title{
Ambiguity in psychology
}

\author{
May, Jon
}

http://hdl.handle.net/10026.1/16456

10.1007/978-3-030-39755-5_4

Palgrave Macmillan

All content in PEARL is protected by copyright law. Author manuscripts are made available in accordance with publisher policies. Please cite only the published version using the details provided on the item record or document. In the absence of an open licence (e.g. Creative Commons), permissions for further reuse of content should be sought from the publisher or author. 
Ambiguity in psychology

Professor Jon May

School of Psychology, Cognition Institute

Plymouth University

Drake Circus, Plymouth PL4 8AA

Jon.may@plymouth.ac.uk

http://www.plymouth.ac.uk/staff/jon-may

ORCID: 0000-0001-7439-9200

Author's copy of Chapter published in S. Popat \& S. Whatley (Eds) Error, Ambiguity and Creativity (2020), Palgrave Macmillan, reproduced with permission of Palgrave Macmillan.

This draft Feb 282019

6799 words 


\section{Ambiguity in psychology}

'Ich stehe am Fenster und sehe ein Haus, Bäume, Himmel. Und könnte nun, aus theoretischen Gründed, abzuzählnen versuchen und sagen: da sind ... 327 Helligkeiten (und Farbtöne). (Habe ich “327”? Nein; Himmel, Haus, Bäume; und das Haben der '327' als solcher kann keiner realisieren.)'

'I stand at the window and see a house, a tree, the sky. And I can, theoretically, try to count up and say: there are ... 327 patches of light (and shades of colour). (Have I got '327'? No: sky, house, tree; and the 327 as such cannot be realised.)' (Wertheimer, 1923).

How do we come to experience houses, trees, and the sky as discrete entities in the world when our senses provide us with partial, fragmentary and sometimes contradictory sources of information, made up of hundreds of patches and shades of light; tones and intensities of sound; or the edges, corners, curves and textures of touch? It is not obvious how we can arrive at a certain, immediate and unambiguous perception of the world around us, given such ambiguous and separate sensory details. The resolution of ambiguity turns out to be something our minds are specialised in, and ambiguity has proven to be of central importance in psychology, from understanding perception to the understanding of creativity and psychological applications in design.

Max Wertheimer was a psychologist who, along with Kurt Koffka and Wolfgang Köhler, helped to found Gestalt psychology in the early years of the $20^{\text {th }}$ century. At that time, psychology was dominated by a method called introspection, in which trained observers would try to report in finer and finer detail the qualities and characteristics of their perception of phenomena. Their goal was to identify similarities and differences between the moments that they were experiencing, across all forms of sensation. The Gestalt psychologists disagreed with this approach, arguing that the whole form being experienced was different to its parts, and it is this that Wertheimer is emphasising in the quotation, taken from his contribution to a festschrift for his old Professor from Berlin, Carl Stumpf.

All perception can be seen as the resolution of ambiguity. When we listen to someone speaking, the words they wanted to say have been turned by their mouths and tongues into pulses of air that spread out and arrive at our ears as rapid variations in air pressure, combined with all of the other sources of 'noise' in our environment. Somehow, we hear in our heads the words that they said - at least, we hear what we think they wanted to say. 
Vision is even more miraculous. If we know a little about the eye, we may think of it like a camera: it has a lens that focuses light onto the retina, which captures the image, and that is what we see. In fact, this is only part of how the eye works. The lens does a good job of focussing light, but there the similarity with a camera ends. Instead of transparent air, the eye is filled with a gel that can contain dead blood vessels and detached fibres that are sometimes experienced as annoying 'floaters' or blobs in our vision, especially after middle age and in the short-sighted. The retina itself is composed of over 100 million cells that are sensitive to different wavelengths of light. Compared to the 12 million pixels that may make up a typical digital photograph, this sounds good, but the before it hits the retina the image has to make its way through a layer of tissue containing a network of blood vessels, feeding the photoreceptive cells. If you take your camera and cover the lens with red tissue paper, you'll get the idea.

Even when the image has made it through to the retina, $95 \%$ of the cells are the rod cells that just detect light intensity or brightness, responding most strongly to a bluish-green light that corresponds to daylight. Just 5\% are the cone-shaped cells that are specialised for blue, green or red light. In dim light, these less sensitive cone cells cannot capture enough light to fire, limiting our vision to the highly sensitive rod cells, and our twilight world becomes largely monochromatic.

But that is not where the image we see comes from. The photoreceptor cells send their information not to a huge 100 Megapixel canvas, but to a complex series of around a million ganglion cells that collect the inputs from a circular range of adjacent rods and cones, and are specialised in detecting patches of colour and movement. These then feed across the brain to the visual cortex at the back of the brain - where, of course, the image from the other eye is also being received and the two have to be reconciled, by cortical cells that combine the outputs of many ganglion cells to detect edges and areas and even shapes. If there is an image anywhere, it is in the visual cortex, where the cells are spatially laid out to represent the visual field, albeit in a 'fish eye lens' format, with the centre of our gaze swollen and the periphery reduced. Damage to the cortex here can lead to selective blindness in the corresponding part of the visual field - although the individual remains perceptually unaware of the damage, in the same way that we are all unaware of the blind-spot in our retina, where a hole for the optic nerve means that no photoreceptors can capture a small part of the image.

This sequence of abstraction of information from a noisy image has led vision researchers to focus on understanding the 'bottom-up' way that details necessary for vision can be extracted 
or computed from the raw image, and upon the connections and sequence of neural operations required to achieve the apparently vivid and clear perceptual scene from the translucent, blood-obscured retinal image. This computational phase can take a blobby or fuzzy pattern and produce a sharp edge between two coloured areas, moving in a particular direction at a steady speed, or with a certain acceleration. Put all of these edges together and we have vision. But there needs to be a great deal of processing and interpretation in this phase. Sometimes the input provided by the photoreceptors and summed by the ganglion cells and analysed by the cortex could have been caused by any one of a range of 'real' sources out in the world. Let's not even bring in the problem that the light around us changes direction and frequency range throughout the day, that our eyes move around, and that we see objects from different orientations and that they move and change shape yet still remain 'objects'. In other words, what we get is not at all what we see.

The Gestalt psychologists were trying to point this out a century ago. Today they are best remembered for the 'laws of grouping', some organisational principles that tried to identify when elements or parts would be perceived as belonging to a larger unit - in German, literally the Gestalt. Wertheimer's 1923 paper contains 56 examples illustrating different ways in which parts form wholes. Some of these are still referred to in textbooks on design, with names such as similarity, proximity, symmetry, order, continuity, closure and figure/ground. Taken at its broadest, parts that in some way share 'common fate' or resemble each other in some way, tend to be grouped together. Where this becomes challenging, and indeed, ambiguous, is when different ways of grouping parts allow more than one possible organisation to be imposed upon them. For example, if you have drawn a series of dots, equally spaced horizontally, they will be perceived as a line (Fig 1a). Even if they are not all horizontal, but weave up and down as they cross the page, they form a curving, irregular gestalt (Figure 1b). The mind conceptually fills in the gaps between them to join them together - the gaps are still perceived, and so are the dots, but then so is the line. But where is the line? It isn't there on the page, and it hasn't been 'sensed': the gaps are still there. The line is in the mind: it has been perceived, if not sensed.

This important distinction between perception and sensation shows that the completely bottom-up idea of vision - and of other forms of sensation - is not the whole story. Our minds use the sensory input but create a perception from it by imposing order. The Gestalt psychologists wanted to identify principles that would apply to all sensation, so that this construction could be predicted and understood. They saw the completion of wholes from the parts as a process that was driven by the stimulus, the sensation. 
Look again at a row of dots, and now move every second dot slightly to the left (1c). Immediately, a sequence of pairs has appeared; still a whole sequence, but now with a substructure. Move the right hand dots downwards, and there is still a horizontal row of pairs (1d). Move them further down, and then to the right. At some point, the 'pairs' break up and you may see a zig-zag line, or two separate horizontal lines (1e). Continue moving the dots down, and a zig zag becomes untenable, and the perception of two lines dominates (1f). As these dots moved around, different organisations became more or less likely. When one was supported strongly by one or more principles, and no others were, then only that organisation was perceived. When different principles suggested alternative organisations, then both might be tenable, and the pattern can be said to be ambiguous. Interestingly, though, while you can flip between two or more organisations, seeing in Figure 1e either a zig-zag or two straight lines, you cannot perceive both organisations at the same moment. Such patterns are said to be bistable, as they can be stably perceived in two ways.

Auditory examples also exist - instead of dots, imagine notes being played on a piano. Play A repeatedly, at a steady pace: A---A---A---A---. Now play C between each A: A-C-A-C-A-CA-C-. Here we have an auditory analogue of the zig zag line. Now bring the $\mathrm{C}$ closer to the A: AC- -AC- -AC- - AC- - and you can hear a single sequence of pairs. Perhaps you can drop the $\mathrm{C}$ a couple of octaves, and in $\mathrm{A}-\mathrm{C}_{2}-\mathrm{A}-\mathrm{C}_{2}-\mathrm{A}-\mathrm{C}_{2}-\mathrm{A}-\mathrm{C}_{2}$ - hear two 'rows' of notes. If you play the As on a piano, and the Cs on a guitar or a violin or a flute, then it becomes easy to hear two sequences of notes. If two instruments are playing series of notes that are not related, then we perceive them separately; when they are related by some principle of similarity or common fate, then we perceive a single melody or tune.

Initially the Gestalt idea was that the principles underlying grouping were a structural part of the perceptual system, and that they operated automatically, in much the same way that we now know retinal ganglions combine the inputs of photoreceptor cells to detect patches of colour. A circular line whose endpoints did not quite meet was perceived as 'a circle (with a gap)' because - somehow - perceptual 'energy' could flow across the gap to fill it in. While this makes some sense in modern terms, with what we know about neural networks providing 'best fit' outputs for noisy inputs, at that time there were no convincing explanations for how closure or completion could actually occur in the brain.

An indication that the mind was involved rather than the brain came with the discovery of more complicated bistable images, in the form of ambiguous images. The most famous of these were not designed by the psychologists, but had existed for decades as puzzle pictures. Perhaps the most famous is the duck-rabbit, first published in a German magazine in 1892 
(Figure 2), with the caption 'Which animals are most alike? Rabbit and duck.' This picture was described by Joseph Jastrow in 1899, who noted that 'most observers find it difficult to hold either interpretation steadily, the fluctuations being frequent, and coming as a surprise', and later fascinated Wittgenstein, who included it in his 1953 Philosophical Investigations.

In 1930 Edwin Boring described an 1888 postcard (Fugure 3) depicting 'my wife and motherin-law' as 'superior to most of the better known puzzle pictures, because of the fact that neither figure is favoured over the other, although both figures interpenetrate the same region of the total field.' (se McManus et al., 2010)

These illustrations are fascinating because it is difficult to believe that there can be perceptual principles involved in identifying rabbits, ducks or women of different ages, and yet the different interpretations seem to come to the fore spontaneously, and each is convincingly real, and incompatible with the alternative, which can no longer be seen (Chambers \& Reisberg, 1992).

The original puzzle pictures are highly representational, with shading and hatching to give a sense of a solid form, although more stylised forms have been developed that strip away the superfluous details. Wittgenstein's version of the duck-rabbit, for example, was a simple line drawing with a single dot for an eye. Perhaps crucially, in both images the 'nose' of the rabbit and the young woman need to remain as a ruffled feather at the back of the duck's head, and a pimple on the old woman's nose, for their interpretations to become possible. In return, the young woman wears a necklace (which becomes the old woman's narrow mouth), although the duck contains no unnecessary bits of rabbit.

A much more stylised bistable figure had been discovered some time earlier, in 1832 by a crystallographer, Louis Necker, who was looking with one eye through a microscope at transparent cubic crystals. With just one eye, he could not tell which face of the cube was at the front and which was at the back. One moment he would be sure that one was at the front, and then it would instantly be at the back. Even worse, with each change in position of that face, the orientation of the whole cube would seem to flip dramatically. Today, Necker's cube is familiar as a wire-frame drawing, and has been used much more widely in studying perceptual bistability than any of the other more representative figures. Studies of brain waves have found signals suggesting that changes in activity occur throughout the perceptual system, leading to the conclusion that if top-down influences are driving the switch in perspective then they can have a very pervasive influence at remarkably early levels of perception, a few tens of milliseconds into processing (Palmer, 2002; Kornmeier \& Bach, 2005). 
A challenging finding was reported by Deborah Chambers and Dan Reisberg in 1985. They showed people the duck-rabbit figure for five seconds, then took it away, and asked their volunteers to imagine the images. While inspecting these mental images, people did not report any reversals, but just reported one stable interpretation. The volunteers then drew their images onto paper, and as they inspected what they had drawn, experienced flipping again. Chambers and Reisberg (1985) argued that mental images were therefore entirely conceptual and lacked the geometric or low-level perceptual content that was necessary for alternative representations to be possible. While this idea is appealing, it raises the difficult question of what form the concepts have, if they subjectively feel like 'pictures in the head' but lack pictorial content.

One answer to this comes from a representational model of mental processing proposed by Philip Barnard, called Interacting Cognitive Subsystems, or ICS (Barnard, 1985). Originally this model was developed in the 1980s to explain the way that people inferred information when listening to narratives or reading text, but has since been applied in a variety of contexts including clinical psychology (to describe the interaction of emotional and rational thoughts), computer interface design (to predict the problems people have when the design does not match the task they have in mind), and creativity (helping people develop strategies to use their imagination effectively).

ICS sees the mind as a set of systems, each specialised to store and process a different level of mental representation (Figure 4). The sensory levels deal with acoustics, vision and the body, respectively. Additional Effector levels control large-scale skeletal bodily movements, somatic and visceral responses, and detailed vocal articulation needed for speech, respectively. Linking the sensory and effector systems are four central systems that receive output from the sensory systems, exchange representations amongst themselves, and produce outputs for the effector systems.

The job of the three sensory systems is to combine information about the world from the ears, the eyes and the more physical contact senses of touch, smell and taste, and to produce abstract schematic representations for the central systems to process. One of the central systems deals with spatially organised objects, receiving information from the visual sensory system; another with sound sequences recognised by the auditory sensory system. These object and sound systems hold information that is more clearly perceptual than sensory, and correspond to our subjective mental imagery. The physical sensations derived from contact are combined into a holistic representation of their emotional and affective implications primarily whether they are pleasant or to be avoided, but also whether they are threatening or 
signal safety. Crucially, all three of these central levels produce an output that forms the fourth level - they can identify or label their contents in a propositional form. This final system therefore potentially combines the sounds, objects and implications into a factual description of the world. In turn, it provides inputs to the other central systems, linking them together in reciprocal loops.

When we see an ambiguous visual figure like the duck-rabbit, the sensory information is first processed by the visual subsystem. Its object-level output is still ambiguous, and is not yet identified with the label of duck or rabbit - that labelling is performed by the central object subsystem, and the label is propositional in form. If you had never seen this figure before, then the object subsystem would base its output on the similarity to other drawings stored in its long term memory. The more closely the figure matches your personal experience of recognising similar images of ducks, the more likely you are to identify a duck. The closer it is to your experience of recognising similar images of rabbits, the likelier you are to see a rabbit. Your propositional system then receives the label 'duck' or 'rabbit' and immediately sends more relevant information to the object level - helping to strengthen the initial interpretation, so it now becomes much more clearly a duck or rabbit. If at this point the image were taken away and you were asked to draw what you had seen, your drawing would resemble a canonical duck or rabbit, more duck-like or rabbit-like than the actual image.

This sequence by which we impose a meaning upon our sensations and then perceive what we have identified the sensations as representing was demonstrated in the 1930s by Frederick Bartlett (Bartlett, 1932), who showed people abstract geometrical drawings, and then asked them to draw them again from memory. Usually people would recall the forms fairly accurately, but not completely photographically - they would straighten things, make them more symmetrical and regular, and omit a detail or two. He then showed these new drawing to a second person, and asked them to draw it from memory. And so on. As this sequence progressed, the drawings became more and more stylised and regular, as successive people made 'corrections' and dropped inconsistent details, until the final drawing bore only a faint resemblance to the original.

If you have ever played Chinese Whispers, you will recognise a similar process through with muffled or nonsensical sounds have more regular meanings imposed upon them as they pass from person to person. Bartlett then repeated the experiment by asking individual people to recall the image, over time. From day to week, and week to month, their drawings showed the same tendency to become more and more regularised, as the original sensory memory became dominated more and more by the propositional labelling imposed at each recollection event. 
He also asked people to read and then recall a folk story from a different culture, finding not only that they had great difficulty doing so but that their recollections became shorter and more 'comprehensible' as they imposed their own meanings upon the material. In all these cases, Bartlett felt that information was being encoded in the mind using schemas - consistent patterns or stories that had been acquired through experience over time. The schema was also used to recall and reproduce the information on demand. Where information did not fit a schema, it was not encoded, not stored, and not recalled. In its place, schematically congruent information would be invented to join otherwise unrelated pieces of the memory.

Bartlett's work on schemas shows that memory is reconstructive, and Barnard's model shows that perception is interpretive, with our expectations influencing our subjective experience of the perceptual world. When sensory information accords with a particular interpretation, the feedback loop between the object and propositional levels rapidly stabilises the percept upon the appropriate form, inconsistent sensory information is ignored (although it is still experienced and can be reported while the stimulus remains to be sensed) and missing information is inferred. When sensory information is ambiguous, the feedback loop again imposes a single interpretation and for some moments the perception is clearly of a particular interpretation. As long as the feedback loop remains active, with the object and propositional levels supporting each other, this interpretation remains stable.

The mind, though, is never doing just one thing. There are always competing demands upon processes and the propositional level of representation is involved in many potential flows of information through the overall cognitive architecture. If it engages for a moment in another task, dealing with sound or implicational information, the feedback loop is interrupted and the object level receives only the uninterpreted output from the sensory level. This provides a chance for the alternative perception to take hold; as the propositional system has no link directly with the sensory system it now receives a different input and reinforces that interpretation instead. As far as it has concerned, the perceptual world has altered, and we experience that strange shift. The duck becomes a rabbit, although at the object level we can tell that the sensory input has not changed.

Barnard's conception of multiple simultaneous levels of representation interacting within our minds helps to resolve the paradox of how we can experience the identity of an object changing while knowing that it has not changed to our senses. It also deals with the far more common and less spooky experience of the sensory information changing but an object's identity remaining constant - in fact we need the stable internal representation to make sense of an inconsistent world. We seldom have to make sense of things that pop into our sensory 
world without warning, but far more typically sense things within an ongoing context that helps us to recognise them.

Distraction or interruption is not the only opportunity for a stable interpretation of an ambiguous figure to alter: we can impose a propositional alteration at will and by concentrating upon, say, the rabbit-perspective force a duck to become a rabbit. With the Necker cube, the foreground face can be pushed to the back, or the orientation of the cube to change from pointing 'top right' to 'lower left'. A similar feedback loop between sound and propositional representations shows how we can switch between different interpretations of bistable auditory stimuli, and the interpretation of ambiguous sentences such as 'the pirates opened the dead man's chest' - here the (perhaps unpleasant) visual images that come with the competing interpretations also play a supporting role, showing that the whole cognitive system can come into play to support disambiguation.

In clinical psychology, the loop between propositional knowledge and its implicational interpretation is important in understanding that what might seem to a depressed person to be a completely rational negative evaluation of their life can be seen as completely irrational to their family. The true value of ambiguous figures is that they show us how the mind is always constructing an unambiguous reality from the ambiguous information that is available to it, in the short term for small stimuli but in the longer term too as we build an understanding of our own lives.

Gestalt psychology faded as the twentieth century progressed and the behavioural psychologists dominated in the post war years, with their explicit rejection of introspection and a reliance on measuring behavioural responses that could be observed. The behaviourists sought to determine laws of association between stimulus and response that needed no role for mental representations as mediating constructs. If a stimulus was ambiguous, in that it produced a mixed pattern of responses, it was just that a dominant response had not been learnt sufficiently.

It did not take too long for ambiguity to play a key role in reasserting the mind's importance, though. From the 1960s psychologists returned to the Bartlett's concept of schemas to explain how people could apparently jump to conclusions and learn some associations after only one presentation, far too quickly for the probabilistic laws of learning to cope with. Schemas also made sense of experiments where the context of the information was essential. In one famous experiment in 1972, John Bransford and Marcia Johnson asked people to read a paragraph starting with the following rather ambiguous material: 
'The procedure is actually quite simple. First you arrange things into different groups. Of course, one pile may be sufficient depending on how much there is to do. If you have to go somewhere else due to lack of facilities that is the next step, otherwise you are pretty well set. It is important not to overdo things. That is, it is better to do too few things at once than too many. In the short run this may not seem important but complications can easily arise. A mistake can be expensive as well. At first the whole procedure will seem complicated...' (Bransford \& Johnson, 1972, p.722)

Given this text alone, people rated their comprehension very poorly, at around two out of seven. In a recall test, they could only remember just under three pieces of information. If before reading the text people had been told 'the paragraph you will read will be about washing clothes', then things changed dramatically. These people rated their comprehension at 4.5 out of 7 , and on average recalled almost six of the pieces of information. The contextual information had, according to the experimenters, allowed people to use a schema about laundering to understand the information as they read it, and so to disambiguate it.

A third group read the text first and were then given the information 'it may help you to know that the paragraph was about washing clothes', but their comprehension and recall was no better than the first group, showing that the schematic information needed to be active while the information was being read if it was to help: on its own, the schema did not help recall. Without schematic information being available to support inference about the paragraph while it was being read, recall of the text could only be based upon the words themselves - in Barnard's terms, there could be no top-down structuring or interpretation of the material from propositional or implicational representations. If you ever had to learn something by rote in school, then you will know how difficult it can be, and that while you may eventually learn to repeat the words red, yellow, green, blue, orange, indigo, violet in the right order doing so does not help you understand refraction and reflection of light by water droplets. Adding some irrelevant schematic information by making a meaningful mnemonic from the initial letters (Richard of York gave battle in vain) may speed up the learning, but still does not help with understanding why the colours of a rainbow are in that particular order.

Schematic knowledge is very useful in our day to day lives and pervades our normal thought processes, helping us to resolve the ambiguity that is inherent in almost every situation. Barnard has argued that human thought is unique because we alone amongst animals possess the propositional level of representation. The labelling and abstraction that this provides supports schematic inference through its feedback loops with the other three central systems. 
Other animals have to rely on loops involving the holistic, implicational level, and so their inferences are more tightly linked to sensory and emotional information. While schematic inference is enormously useful in most contexts, it does have some limiting aspects, and another of the areas studied by Gestalt psychologists demonstrate this - creative problem solving.

Gestalt psychologists were especially interested in the sort of problems that could not be solved by working through a well practiced procedure or routine, but which appeared to require a flash of creative insight. For example, Karl Duncker invented a problem in which people were shown pictures of a candle and a box of tacks, and asked to fix the candle to the wall to that it could be lit without the wax dripping onto the floor. Any attempt to tack the candle directly to the wall would fail because the wax was too brittle. The solution required people to realise that the box holding the tacks could itself be tacked to the wall as a shelf, and that the candle could then be balanced in it.

Duncker's problem is hard because we use schematic information and see the items we are given in terms of their conventional uses. The box is just there to hold the tacks. To solve the problem we have to put aside the conventional schematic labelling and see the box as part of the solution. Restructuring the problem like this allows all of the other schematic information about the box to come into play and the solution hits us all at once and we have an 'Aha!' moment. The Gestalt understanding of these problems was that the conventional representation formed quickly through inference and analogy with schematic knowledge of the problem elements, and that this 'functional set' had to be put aside to allow more unusual uses to be considered. In other problems, 'procedural set' might lead to someone applying a familiar but inappropriate routine when a novel routine was needed.

Sometimes, restructuring the problem required seeing the materials in a novel way. The mutilated chessboard problem proposed by Max Black in 1946 asked if it were possible to cover a chessboard with 31 dominoes, when the two opposite corners had been removed, leaving 62 squares. Each domino was big enough to cover two adjacent squares. Trying to solve this problem practically, laying out the dominoes in different ways, is futile, because the answer is simply 'No'. While many people suspect that this is indeed the answer, but cannot prove it, it becomes obvious if three aspects of the problem are pointed out to them. First, the squares on a chessboard alternate in colour, and you never get two black or two white squares together: this point is obvious and people do not need to be told it, or think it helps, but it does. The second aspect is that each domino covers one square of each colour-again, this is obvious, and while people might not think about it until it is pointed out to them, they accept 
it readily. Less obviously, the third point is that the two corner squares diagonally opposite each other on a chessboard are always of the same colour - both black or both white.

Removing them leaves 32 of one colour and 30 of the other.

As soon as one has this final point, it is easy to see that once you have lain down 30 dominos covering 30 squares of each colour, you will be left with two identically coloured squares, which cannot be adjacent to each other, and so cannot be covered by the single remaining domino (unless, creatively, you snap it in half). Solving the problem requires the chessboard to be seen not as a patterned surface to be tiled, but as a collection of individual squares with an alternating arrangement. Even calling it by a different name, such as 'bread and butter board', with alternating squares of bread and butter, helps to overcome the conventional ideas and make the identical nature of the opposite corners more apparent.

It is arguably these problems' lack of ambiguity that makes them difficult. We know a box is a box, and a chessboard is a chessboard, and have to overcome these schematic labels and see them in an unusual way to solve the problem. Making the box or the chessboard less familiar - more ambiguous - helps with problem solving because it stops us using our inferred information-as-usual approach and forces us to use the more limited information that is in the problem itself.

Research into creativity has emphasised that coming up with unusual uses for objects is a hallmark of the creative person. The most well-known test of creativity, developed by J.P. Guilford in 1960, is even called the 'Alternative Uses' task, and asks people to think of as many uses that they can think of for an everyday item such as a brick. While the first answers that people come up with are the obvious ones, after these are exhausted less usual answers are produced, and so there is a direct relationship between the number of responses you can produce in a given time and their originality. The ability to generate unusual ideas has been called divergent thinking, as one's ideas get further and further away from the first, schematically constrained, ideas. In contrast, ways of thinking that circle around the conventional are called convergent.

Unusual uses are important because they offer the potential for us to see the elements of a problem in the new way that may be needed to come up with a creative solution or application for them. It is not the whole story, of course, because not every unusual use or viewpoint is going to be useful. In 1926, Graham Wallas pointed out that the divergent 'illumination' part of creative problem solving, where the solution appeared as if in a flash of insight, had to be followed by a more convergent 'verification' phase in which the answer was checked out. 
Before illumination could occur, he also listed a 'preparation' phase where the problem had to be defined and understood, and all of its elements identified, followed by an 'incubation' phase, where you didn't actively think about the problem. This paradoxical part of problem solving - not doing anything - may seem rather zen-like, but it makes sense if you see it as a time to let the schematic ideas about a problem fade away, giving a chance for the unusual ideas to make themselves heard.

Presenting people with problem related material in ambiguous ways, or making the material more ambiguous, might help with the incubation phase of creative problem solving, by making the schematic inferences less apparent, and strengthening the unusual. Wallas' framework suggests that there is a fine line to tread though, because many of the unusual uses or ambiguously presented parts may not be the appropriate ones to generate a solution - many paths may diverge from a schema without reaching a useful goal, and offering too many or too diverse a set of alternative representations might make the useful one harder to find, increasing the difficulty of the verification phase.

Working out how best to make something helpfully ambiguous seems like a daunting task, as surely you have to know what the solution is going to be to know which parts of the problem ambiguous. Making everything about it ambiguous is likely to set people onto unprofitable trails, diverging away from the conventional but getting no nearer to a useful answer. This is where an analysis of the general type of problem, using Barnard's ideas about levels of representation, might help. Identifying which levels of representation that are involved when normally tackling a particular type of problem can indicate which of the four central levels is providing the schematic information, and so how it can be made less dominant. For example, if we thought that parts of a problem were being named and then canonical ideas about those items were being used to generate solutions, we might try to make the canonical ideas less influential by making the parts harder to name - making their identities more ambiguous. If the canonical functions of the parts are interfering, then making those functions less obvious should help - so in Duncker's candle problem, showing people the tacks and the box separately removes the proposition 'box is here as a container' and makes it more likely that they will see it as something to be used in the solution.

In general, making the mappings from the sensory visual and acoustic information to the structural object and sound levels more ambiguous just makes common forms harder to identify propositionally, and although we are highly practiced at disambiguating such stimuli it may give our structural subsystems the opportunity to misidentify something, or to strengthen a potential alternative labelling. Whether or not this is useful in coming to a novel 
use of the material is then down to chance and the likelihood that the misidentification is still going to be contextually influenced by the top-down problem-relevant stream of information from the propositional level that is constraining the range of things one is likely to be seeing. Leaving the sensory information unambiguously clear, but presenting the objects or sounds in an unusual orientation or voice, or altering their visual or sonic context, would more directly influence their propositional labelling, and the very unusualness of their presentation would also be identified and help to weaken any canonical associations: if a box is not holding anything, then the idea that 'boxes are just containers' is not give as much support as it is when it is full of tacks. Altering the emotional or contact-sense information about part of the problem will help to defeat any implicational constraints upon its identification or use, and therefore promote novel application such as, for example, combining food ingredients in different ways to create new culinary experiences; or changing the emotional context might help someone to infer a novel meaning to a conversation or message and so see an interpersonal conflict in a new and more constructive light.

Ambiguity is something we deal with all of the time, usually by resolving it and prioritising the familiar and most likely interpretations. While for most purposes, these are exactly what we need, they can constrain us when we are faced with novel problems, unusual situations, or tasks for which experience is less relevant than novelty. In these contexts, we can be helped by increasing ambiguity, either in the materials we have to hand, or in our own mental approach, so that the mundane can be replaced by the extraordinary. 


\section{References}

Barnard, P.J. (1985). Interacting cognitive subsystems: a psycholinguistic approach to short-term memory. In A. Ellis (Ed.), Progress in the psychology of language, Vol. 2. Hove, UK: Laurence Erlbaum Associates Ltd.

Bartlett, F.C. (1932) Remembering: An Experimental and Social Study. Cambridge, UK: Cambridge University Press.

Bransford, J.D. \& Johnson, M.K. (1972) Contextual prerequisites for understanding: some investigations of comprehension and recall. Journal of verbal learning and verbal behaviour, $11,717-726$.

Chambers, D. \& Reisberg, D. (1985). Can mental images be ambiguous? Journal of Experimental Psychology: human perception and performance, 11, 317-328

Chambers, D. \& Reisberg, D. (1992). What an image depicts depends on what an image means. Cognitive Psychology, 24, 145-174.

Guilford, J.P., Christensen, P.R., Merrifield, P.R., and Wilson, R.C., (1960). Alternative Uses Manual. Sheridan Supply Co.

Kornmeier, J. \& Bach. M. (2005) The Necker cube - an ambiguous figure disambiguated in early visual processing. Vision Research, 45, 955-960.

McManus, I.C., Freegard, M., Moore, J. \& Rawles, R. (2002) Science in the making: right hand, left hand. II: The duck-rabbit figure. Laterality, 15, 166-185.

Necker, L.A. (1832) Observations on some remarkable optical phænomena seen in Switzerland; and on an optical phænomenon which occurs on viewing a figure of a crystal or geometrical solid. The London and Edinburgh philosophical magazine and journal of science, third series, 5, 329-337

Palmer, S. (2002) Perceptual Grouping: It's Later Than You Think. Current Directions in Psychological Science, 11, 101-106.

Wertheimer, M. (1923). Untersuchungen zur Lehre von der Gestalt II. Psychologische Forschung, 4, 301-350. 
Figure 1 (a-f) : Gestalt principles of organisation in rows of dots

\section{$\bullet \bullet \bullet \bullet \bullet \bullet \bullet \bullet \bullet \bullet \bullet \bullet \bullet \bullet \bullet \bullet$}

$\bullet \circ \bullet \circ \bullet \bullet \bullet \bullet$

\section{$\bullet \bullet$}

$$
\circ \circ \bullet^{\circ}
$$

\section{0}

$\bullet_{\circ}^{\circ} \bullet_{0}^{\circ} \bullet_{0} \bullet_{0}^{\circ} \bullet_{0}$

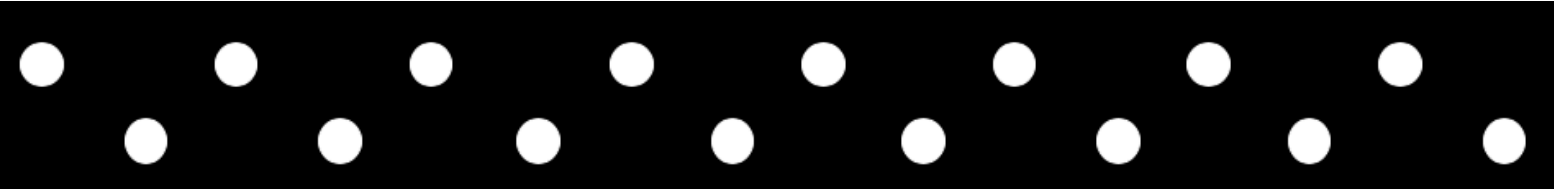

$\begin{array}{llllllll}0 & 0 & 0 & 0 & 0 & 0 & 0 & 0\end{array}$

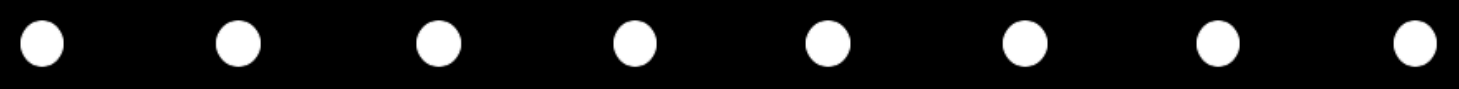


Figure 2: An original 'duck and rabbit' picture

\section{Belde $\mathfrak{a} \mathfrak{n} \mathfrak{d} \mathfrak{r} \mathfrak{a}$ meiften?}

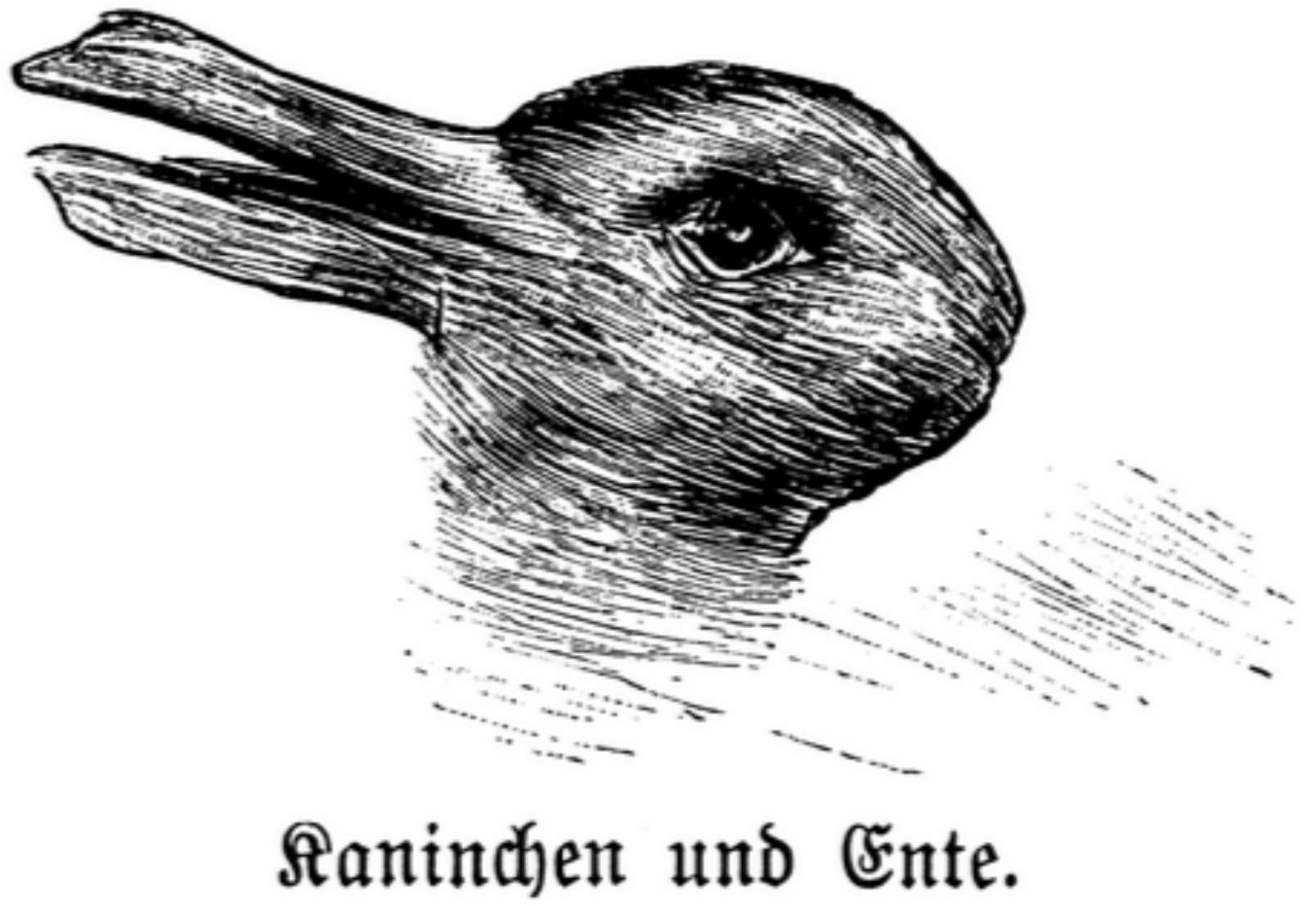


Figure 3: Boring's ambiguous young girl - old woman image

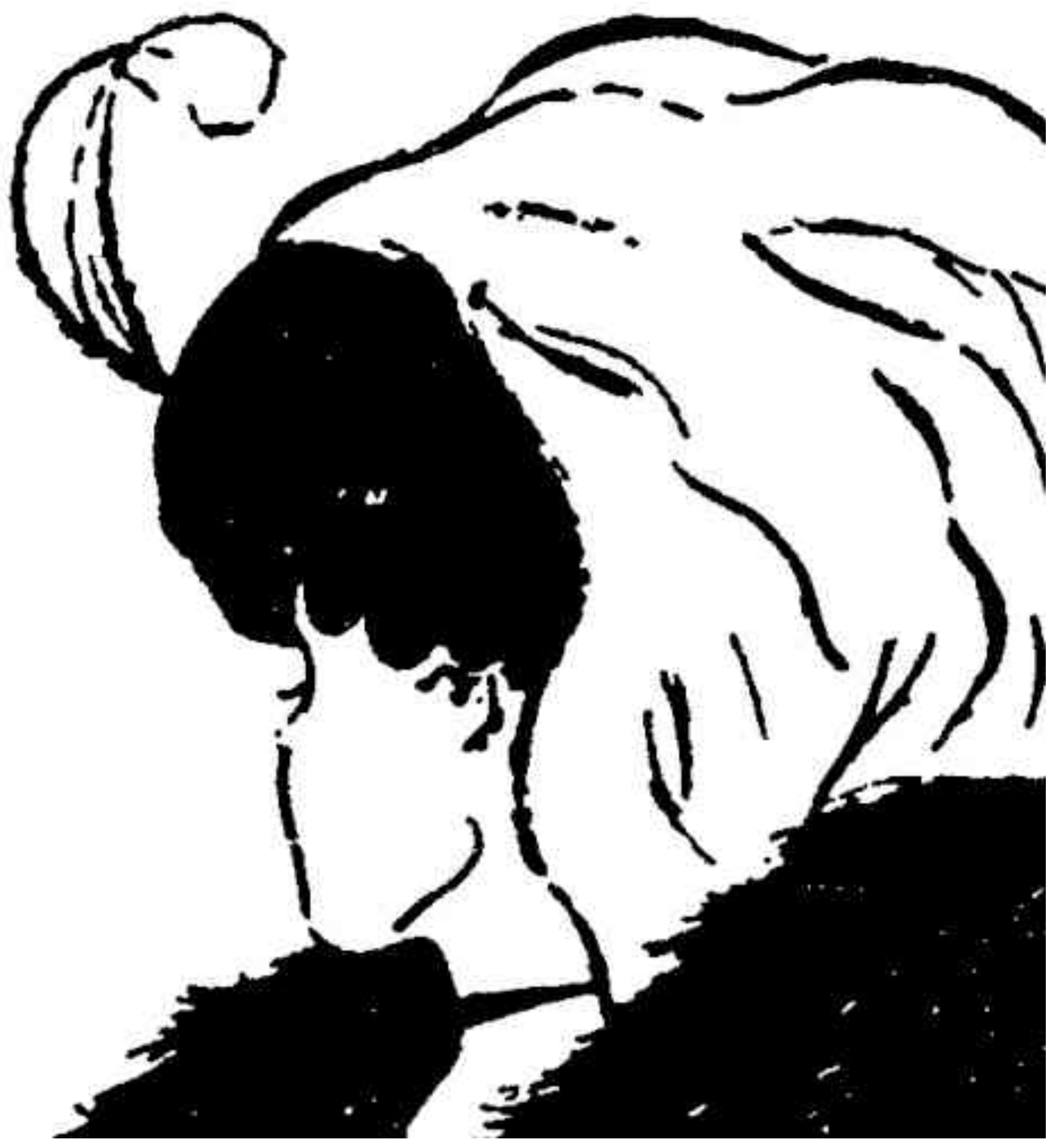


Figure 4: The Interacting Cognitive Subsystems model of mental processing.

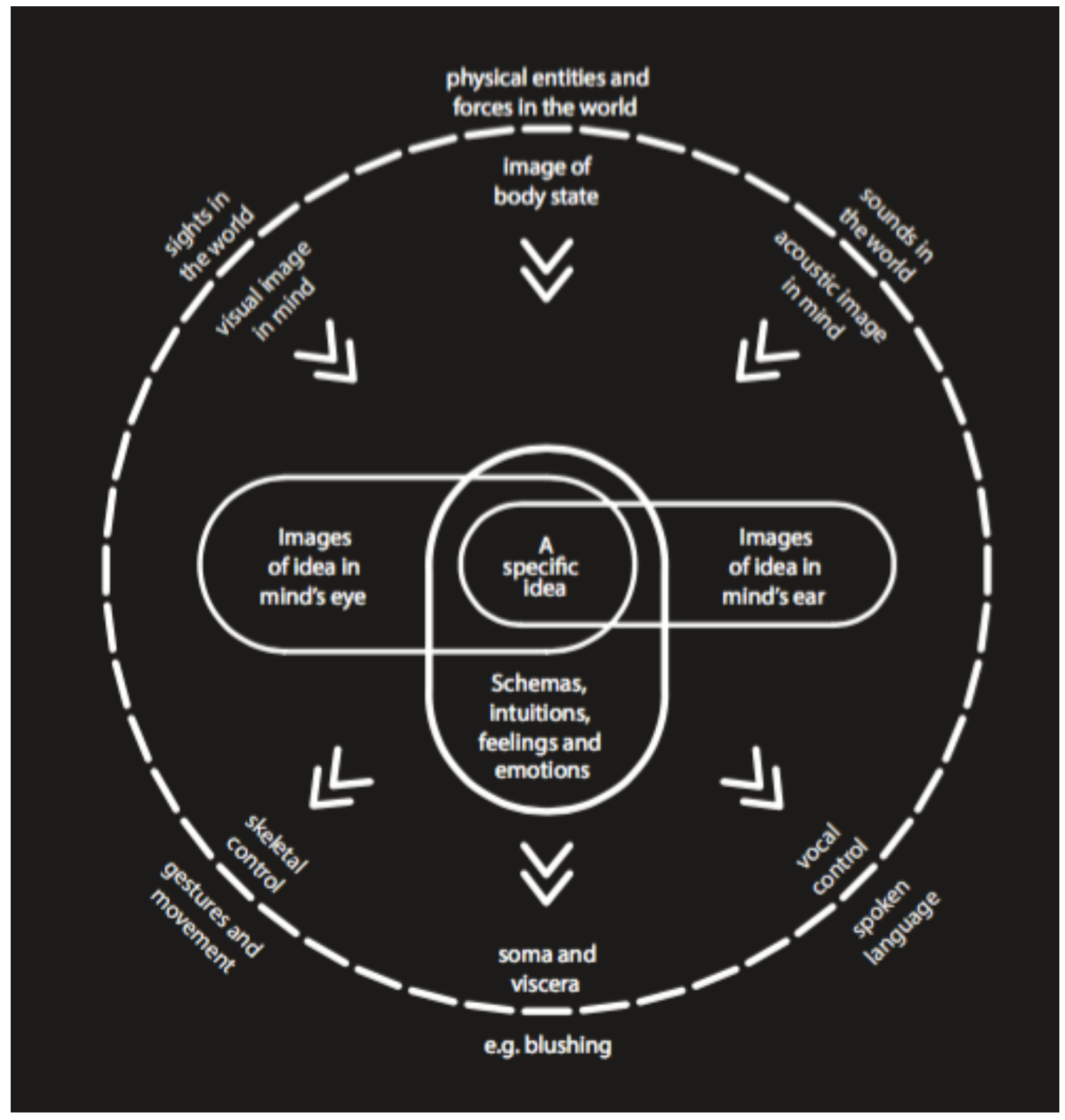

